\title{
Intrabeam scattering formulas for high energy beams
}

\author{
Kiyoshi Kubo, ${ }^{1}$ Sekazi K. Mtingwa, ${ }^{2,3,4, *}$ and Andrzej Wolski ${ }^{4}$ \\ ${ }^{1}$ KEK, High Energy Accelerator Research Organization, Tsukuba, Japan \\ ${ }^{2}$ North Carolina A\&T State University, Greensboro, North Carolina 27411, USA \\ ${ }^{3}$ Harvard University, Cambridge, Massachusetts 02138, USA \\ ${ }^{4}$ Lawrence Berkeley National Laboratory, Berkeley, California 94720, USA
}

(Received 14 October 2004; published 12 August 2005)

\begin{abstract}
We derive completely integrated formulas for emittance growth times due to intrabeam scattering for charged particle beams in the high energy limit, including the effect of lattice parameters that vary around the accelerator ring. Using accelerator lattices for the prototype damping ring called the Accelerator Test Facility at KEK and those for two proposed International Linear Collider damping rings, we compare our results with other calculations.
\end{abstract}

DOI: 10.1103/PhysRevSTAB.8.081001

PACS numbers: 29.27.- a, 41.75.Fr, 41.75.Ht

\section{INTRODUCTION}

Intrabeam scattering (IBS) involves multiple smallangle Coulomb scatterings of charged particles within accelerator beams. This phenomenon leads to the growth in beam emittances, which places severe limitations on luminosity lifetimes in hadron and heavy ion colliders and the ability to achieve ultrasmall beam emittances in intense electron storage rings.

The detailed theory of IBS is described in a number of publications [1-5]. When attempting to use the full theory in many software codes, it takes a long time to compute the dependence of longitudinal and transverse emittances on variables such as elapsed time and bunch charge. Thus, over the years, many authors have attempted to derive high energy approximations to the full theory that are more computationally friendly [6-11]. In particular, Bane [11] has derived a high energy approximation to the theory described in Ref. [4] and has shown the equivalence of that high energy approximation to the high energy limit of a modified form of the Piwinski theory [1].

In the next section, we summarize the general IBS theory for bunched beams contained in Ref. [4] and Bane's high energy approximation to that theory, which we call Bane. In Sec. III, we describe Bane's modification of the original Piwinski theory [1] to include varying lattice functions. Using this modification, we derive a completely integrated modified Piwinski (CIMP) high energy approximation. In Sec. IV, we use the prototype damping ring called the Accelerator Test Facility (ATF) at KEK described in Ref. [12] to compare the Bane and CIMP high energy approximations to each other and to the results obtained using the full theory described in Ref. [4].

Recently, the worldwide high energy physics community decided to pursue a cold, superconducting technology for accelerating structures for the next generation electron-

\footnotetext{
*Permanent address: NC A\&T State University, Greensboro, NC 27411.
}

positron collider. This decision places constraints on the other components of the collider, such as the electron and positron damping rings. The ATF prototype damping ring was dedicated to studying accelerator issues for a warm, normal conducting option for the accelerating structures; however, it is still highly relevant for the superconducting linac option. There have been two recent proposals for damping rings for the cold option: a $6.1 \mathrm{~km}$ circumference damping ring [13], which we call the $\mathrm{ILC}_{\text {small }}$, and the original cold option damping ring [14], which is shaped like a dog's bone, and thus we call it the ILC $_{\text {dogbone }}$ damping ring. In Sec. IV, we compare the Bane and CIMP high energy approximations for all three damping rings by computing the dependencies on bunch charge of equilibrium transverse emittances, relative energy spread, and rms bunch length.

\section{GENERAL IBS THEORY AND BANE'S HIGH ENERGY APPROXIMATION}

For bunched beams, Ref. [4] provides the following general results:

$$
\begin{gathered}
\frac{1}{T_{p}} \equiv \frac{1}{\sigma_{p}} \frac{d \sigma_{p}}{d t}, \\
\frac{1}{T_{h}} \equiv \frac{1}{\varepsilon_{h}^{1 / 2}} \frac{d \varepsilon_{h}^{1 / 2}}{d t}, \\
\frac{1}{T_{v}} \equiv \frac{1}{\varepsilon_{v}^{1 / 2}} \frac{d \varepsilon_{v}^{1 / 2}}{d t},
\end{gathered}
$$

with

$$
\begin{aligned}
\frac{1}{T_{i}}= & 4 \pi A(\log )\left\langle\int_{0}^{\infty} d \lambda \frac{\lambda^{1 / 2}}{[\operatorname{det}(L+\lambda I)]^{1 / 2}}\right. \\
& \left.\times\left\{\operatorname{Tr} L^{i} \operatorname{Tr}\left(\frac{1}{L+\lambda I}\right)-3 \operatorname{Tr}\left[L^{i}\left(\frac{1}{L+\lambda I}\right)\right]\right\}\right\rangle,
\end{aligned}
$$

where $T_{p}, T_{h}$, and $T_{v}$ are the growth times for the relative 
energy spread and horizontal and vertical emittances, respectively, $i$ represents $p, h$, or $v,\langle\cdots\rangle$ indicates that the integral is to be averaged around the accelerator lattice, and

$$
\begin{gathered}
A=\frac{r_{0}^{2} c N}{64 \pi^{2} \beta^{3} \gamma^{4} \varepsilon_{h} \varepsilon_{v} \sigma_{s} \sigma_{p}}, \\
L=L^{(p)}+L^{(h)}+L^{(v)}, \\
L^{(p)}=\frac{\gamma^{2}}{\sigma_{p}^{2}}\left(\begin{array}{ccc}
0 & 0 & 0 \\
0 & 1 & 0 \\
0 & 0 & 0
\end{array}\right), \\
L^{(h)}=\frac{\beta_{h}}{\varepsilon_{h}}\left(\begin{array}{ccc}
1 & -\gamma \phi_{h} & 0 \\
-\gamma \phi_{h} & \frac{\gamma^{2} \mathcal{H}_{h}}{\beta_{h}} & 0 \\
0 & 0 & 0
\end{array}\right) . \\
L^{(v)}=\frac{\beta_{v}}{\varepsilon_{v}}\left(\begin{array}{cccc}
0 & 0 & 0 \\
0 & \frac{\gamma^{2} \mathcal{H}_{v}}{\beta_{v}} & -\gamma \phi_{v} \\
0 & -\gamma \phi_{v} & 1
\end{array}\right) .
\end{gathered}
$$

( $\log$ ) is the Coulomb logarithm to be defined later, $N$ is the number of particles in a bunch, $r_{0}$ is the classical radius of the charged particle, $c$ is the speed of light in vacuum, $\beta$ is the particle speed divided by $c, \gamma$ is the particle energy divided by the rest mass, $\varepsilon_{h, v} \equiv\left(\sigma_{h, v}^{2} / \beta_{h, v}\right)$ are the transverse emittances, $\sigma_{h, v}$ are the rms transverse beam sizes, $\sigma_{s}$ is the rms bunch length, and $\sigma_{p}$ is the relative energy spread. Also, we have the horizontal dispersion invariant $\mathcal{H}_{h}=\left[\eta_{h}^{2}+\left(\beta_{h} \eta_{h}^{\prime}-\frac{1}{2} \beta_{h}^{\prime} \eta_{h}\right)^{2}\right] / \beta_{h}$ and the function $\phi_{h}=\eta_{h}^{\prime}-\frac{1}{2} \beta_{h}^{\prime} \eta_{h} / \beta_{h}$, with similar expressions for the vertical functions. As usual, $\beta_{h, v}$ and $\eta_{h, v}$ are the betatron and dispersion accelerator lattice functions, respectively.

With a suitable change of the integration variable $\lambda$ in Eq. (4), Bane [11] obtains the following high energy approximations:

$$
\begin{gathered}
\frac{1}{T_{p}} \approx \frac{r_{0}^{2} c N(\log )}{16 \gamma^{3} \varepsilon_{h}^{3 / 4} \varepsilon_{v}^{3 / 4} \sigma_{s} \sigma_{p}^{3}}\left\langle\sigma_{H} g_{\text {bane }}\left(\frac{a}{b}\right)\left(\beta_{h} \beta_{v}\right)^{-1 / 4}\right\rangle, \\
\frac{1}{T_{h, v}} \approx \frac{\sigma_{p}^{2}\left\langle\mathcal{H}_{h, v}\right\rangle}{\varepsilon_{h, v}} \frac{1}{T_{p}}
\end{gathered}
$$

where

$$
\begin{gathered}
\frac{1}{\sigma_{H}^{2}}=\frac{1}{\sigma_{p}^{2}}+\frac{\mathcal{H}_{h}}{\varepsilon_{h}}+\frac{\mathcal{H}_{v}}{\varepsilon_{v}}, \\
a=\frac{\sigma_{H}}{\gamma} \sqrt{\beta_{h} / \varepsilon_{h}}, \\
b=\frac{\sigma_{H}}{\gamma} \sqrt{\beta_{v} / \varepsilon_{v}},
\end{gathered}
$$

and there is the final integral to be done

$$
g_{\text {bane }}(\alpha)=\frac{2 \sqrt{\alpha}}{\pi} \int_{0}^{\infty} \frac{d u}{\sqrt{1+u^{2}} \sqrt{\alpha^{2}+u^{2}}} .
$$

Note that Eq. (11) is not useful for calculating $T_{v}$ when the vertical dispersion vanishes.

Now that we have discussed the general IBS theory contained in Ref. [4] and Bane's high energy approximation to that theory, in the next section, we describe the modified Piwinski theory suggested by Bane [11] and do the final integration to yield a result that we call the Completely Integrated Modified Piwinski high energy approximation.

\section{COMPLETELY INTEGRATED MODIFIED PIWINSKI APPROXIMATION}

The standard Piwinski theory of intrabeam scattering is summarized nicely in Ref. [2]. The relative energy spread and transverse emittance growth times are given by

$$
\begin{gathered}
\frac{1}{T_{p}}=A\left\langle\frac{\sigma_{h}^{2}}{\sigma_{p}^{2}} f(\tilde{a}, \tilde{b}, \tilde{q})\right\rangle, \\
\frac{1}{T_{h}}=A\left\langle f\left(\frac{1}{\tilde{a}}, \frac{\tilde{b}}{\tilde{a}}, \frac{\tilde{q}}{\tilde{a}}\right)+\frac{\eta_{h}^{2} \sigma_{h}^{2}}{\beta_{h} \varepsilon_{h}} f(\tilde{a}, \tilde{b}, \tilde{q})\right\rangle, \\
\frac{1}{T_{v}}=A\left\langle f\left(\frac{1}{\tilde{b}}, \frac{\tilde{a}}{\tilde{b}}, \frac{\tilde{q}}{\tilde{b}}\right)+\frac{\eta_{v}^{2} \sigma_{h}^{2}}{\beta_{v} \varepsilon_{v}} f(\tilde{a}, \tilde{b}, \tilde{q})\right\rangle,
\end{gathered}
$$

where $A$ is defined the same as in Eq. (5) and

$$
\begin{gathered}
\frac{1}{\sigma_{h}^{2}}=\frac{1}{\sigma_{p}^{2}}+\frac{\eta_{h}^{2}}{\beta_{h} \varepsilon_{h}}+\frac{\eta_{v}^{2}}{\beta_{v} \varepsilon_{v}}, \\
\tilde{a}=\frac{\sigma_{h}}{\gamma} \sqrt{\beta_{h} / \varepsilon_{h}}, \\
\tilde{b}=\frac{\sigma_{h}}{\gamma} \sqrt{\beta_{v} / \varepsilon_{v}}, \\
\tilde{q}=\sigma_{h} \beta \sqrt{2 d / r_{0}} .
\end{gathered}
$$

The maximum impact parameter $d$ is usually taken to be the vertical beam size and the Piwinski scattering function $f$ is obtained from Ref. [1] as

$$
\begin{aligned}
f(\tilde{a}, \tilde{b}, \tilde{q}) & =2 \int_{0}^{\infty} \int_{0}^{\pi} \int_{0}^{2 \pi} e^{-r\left[\cos ^{2} \theta+\left(\tilde{a}^{2} \cos ^{2} \phi+\tilde{b}^{2} \sin ^{2} \phi\right) \sin ^{2} \theta\right]} \\
& \times \ln \left(\tilde{q}^{2} r\right)\left(1-3 \cos ^{2} \theta\right) \sin \theta d \phi d \theta d r
\end{aligned}
$$

where $f$ satisfies the following relations:

$$
f(\tilde{a}, \tilde{b}, \tilde{q})=f(\tilde{b}, \tilde{a}, \tilde{q}),
$$

$$
f(\tilde{a}, \tilde{b}, \tilde{q})+\frac{1}{\tilde{a}^{2}} f\left(\frac{1}{\tilde{a}}, \frac{\tilde{b}}{\tilde{a}}, \frac{\tilde{q}}{\tilde{a}}\right)+\frac{1}{\tilde{b}^{2}} f\left(\frac{1}{\tilde{b}}, \frac{\tilde{a}}{\tilde{b}}, \frac{\tilde{q}}{\tilde{b}}\right)=0 .
$$


A single integral representation of $f$ was given some time ago by Evans and Zotter [3]

$$
\begin{aligned}
f(\tilde{a}, \tilde{b}, \tilde{q})= & 8 \pi \int_{0}^{1} d u \frac{\left(1-3 u^{2}\right)}{P Q} \\
& \times\left\{2 \ln \left[\frac{\tilde{q}}{2}\left(\frac{1}{P}+\frac{1}{Q}\right)\right]-0.577 \ldots\right\},
\end{aligned}
$$

with

$$
\begin{aligned}
& P^{2}=\tilde{a}^{2}+\left(1-\tilde{a}^{2}\right) u^{2}, \\
& Q^{2}=\tilde{b}^{2}+\left(1-\tilde{b}^{2}\right) u^{2} .
\end{aligned}
$$

To achieve growth times that are similar to those in Ref. [4] in the high energy limit, Bane [11] suggested the following replacements in the Piwinski theory:

$$
\frac{\eta_{h}^{2}}{\beta_{h}} \longrightarrow \mathcal{H}_{h}=\left[\eta_{h}^{2}+\left(\beta_{h} \eta_{h}^{\prime}-\frac{1}{2} \beta_{h}^{\prime} \eta_{h}\right)^{2}\right] / \beta_{h}
$$

which means $\sigma_{h}, \tilde{a}, \tilde{b}$ from this section become $\sigma_{H}, a, b$ from the previous section. For example,

$$
\frac{1}{\sigma_{h}^{2}}=\frac{1}{\sigma_{p}^{2}}+\frac{\eta_{h}^{2}}{\beta_{h} \varepsilon_{h}}+\frac{\eta_{v}^{2}}{\beta_{v} \varepsilon_{v}} \longrightarrow \frac{1}{\sigma_{H}^{2}}=\frac{1}{\sigma_{p}^{2}}+\frac{\mathcal{H}_{h}}{\varepsilon_{h}}+\frac{\mathcal{H}{ }_{v}}{\varepsilon_{v}}
$$

Also,

$$
\tilde{q}=\sigma_{h} \beta \sqrt{2 d / r_{0}} \longrightarrow q=\sigma_{H} \beta \sqrt{2 d / r_{0}} .
$$

To obtain our CIMP, we evaluate the Piwinski scattering function $f$ in Eq. (23), following the procedure described in Ref. [7]. We start with the modified Piwinski expression for $T_{p}$

$$
\frac{1}{T_{p}}=A\left\langle\frac{\sigma_{H}^{2}}{\sigma_{p}^{2}} f(a, b, q)\right\rangle .
$$

For large $\gamma, q$ is much larger than $a$ and $b$. Thus, speaking in relative terms for the size of the arguments of $f$, we have $f$ (small, small, large). It is shown in Ref. [7] that whenever we have $f$ (large, small, large), then the integrals in $f$ can be done. Therefore, following Ref. [7], to convert $f$ (small, small, large) to an expression involving only $f$ 's of the form $f$ (large, small, large), we use the second Piwinski relation contained in Eq. (25). We arrive at

$$
f(a, b, q)=-\frac{1}{a^{2}} f\left(\frac{1}{a}, \frac{b}{a}, \frac{q}{a}\right)-\frac{1}{b^{2}} f\left(\frac{1}{b}, \frac{a}{b}, \frac{q}{b}\right) .
$$

Thus, we now have

$$
\begin{aligned}
f(\text { small, small, large })= & -\frac{1}{a^{2}} f(\text { large, small, large }) \\
& -\frac{1}{b^{2}} f(\text { large, small, large }) .
\end{aligned}
$$

In such a case, where the first and third arguments of $f$ are large compared to the second, Ref. [7] gives in the high energy limit

$$
f(\alpha, \omega, \delta) \approx \frac{-4 \pi^{3 / 2} \ln \delta}{\alpha} g(\omega),
$$

where the Piwinski scattering function $f$ has been reduced to the function $g$, which is given in Ref. [7] by

$$
g(\omega)=\sqrt{\pi / \omega}\left[P_{-1 / 2}^{0}\left(\frac{\omega^{2}+1}{2 \omega}\right) \pm \frac{3}{2} P_{-1 / 2}^{-1}\left(\frac{\omega^{2}+1}{2 \omega}\right)\right]
$$

where $P_{\nu}^{-\mu}$ are the associated Legendre functions. One takes the plus sign for $\omega \geq 1$ and the minus sign for $\omega \leq$ 1 . Note that $g(\omega) \rightarrow \sqrt{\pi}$ as $\omega \rightarrow 1$ from above or below. We have found that the type 3 associated Legendre functions are the correct ones to use. In any event, it is important to check that one can reproduce Table I in Ref. [7] for the function $g$.

We now obtain the emittance growth times for the high energy CIMP approximation

$$
\begin{aligned}
\frac{1}{T_{p}} \approx A 2 & \pi^{3 / 2}\left\langle\frac{\sigma_{H}^{2}}{\sigma_{p}^{2}}\left(\frac{\ln \left(\frac{q^{2}}{a^{2}}\right) g\left(\frac{b}{a}\right)}{a}+\frac{\ln \left(\frac{q^{2}}{b^{2}}\right) g\left(\frac{a}{b}\right)}{b}\right)\right\rangle, \\
\frac{1}{T_{h}} \approx & A 2 \pi^{3 / 2}\left\langle-a \ln \left(\frac{q^{2}}{a^{2}}\right) g\left(\frac{b}{a}\right)\right. \\
& \left.+\frac{\mathcal{H}_{h} \sigma_{H}^{2}}{\varepsilon_{h}}\left(\frac{\ln \left(\frac{q^{2}}{a^{2}}\right) g\left(\frac{b}{a}\right)}{a}+\frac{\ln \left(\frac{q^{2}}{b^{2}}\right) g\left(\frac{a}{b}\right)}{b}\right)\right\rangle, \\
\frac{1}{T_{v}} \approx & A 2 \pi^{3 / 2}\left\langle-b \ln \left(\frac{q^{2}}{b^{2}}\right) g\left(\frac{a}{b}\right)\right. \\
& \left.+\frac{\mathcal{H}_{v} \sigma_{H}^{2}}{\varepsilon_{v}}\left(\frac{\ln \left(\frac{q^{2}}{a^{2}}\right) g\left(\frac{b}{a}\right)}{a}+\frac{\ln \left(\frac{q^{2}}{b^{2}}\right) g\left(\frac{a}{b}\right)}{b}\right)\right\rangle,
\end{aligned}
$$

with $\sigma_{H}, a, b, q$, and $g$ defined in Eqs. (12)-(14), (30), and (34). Whenever there is horizontal and vertical dispersion, the second terms in Eqs. (36) and (37) generally dominate the first terms; thus, one again retrieves Eq. (11), which states that the transverse emittance growth times are proportional to that of the relative energy spread. However, if the dispersion, usually the vertical, is zero or quite small, the first terms in Eqs. (36) and (37) must be kept. Note that the Bane approximation does not include the case of zero dispersion.

We now turn our attention to the Coulomb log factor. Since $q$ is much larger than $a$ and $b$, in the CIMP approximation, we have $\ln \left(q^{2} / a^{2}\right) \approx \ln \left(q^{2} / b^{2}\right)$, so we can pull the common factor of $\ln \left(q^{2} / a^{2}\right) \equiv(\log )$ outside all the expressions. Finally, we obtain

$$
\frac{1}{T_{p}} \approx 2 \pi^{3 / 2} A(\log )\left\langle\frac{\sigma_{H}^{2}}{\sigma_{p}^{2}}\left(\frac{g(b / a)}{a}+\frac{g(a / b)}{b}\right)\right\rangle,
$$




$$
\begin{aligned}
\frac{1}{T_{h}} \approx & 2 \pi^{3 / 2} A(\log )\left\langle-a g\left(\frac{b}{a}\right)\right. \\
& \left.+\frac{\mathcal{H}_{h} \sigma_{H}^{2}}{\varepsilon_{h}}\left(\frac{g(b / a)}{a}+\frac{g(a / b)}{b}\right)\right\rangle, \\
\frac{1}{T_{v} \approx} & 2 \pi^{3 / 2} A(\log )\left\langle-b g\left(\frac{a}{b}\right)\right. \\
& \left.+\frac{\mathcal{H}_{v} \sigma_{H}^{2}}{\varepsilon_{v}}\left(\frac{g(b / a)}{a}+\frac{g(a / b)}{b}\right)\right\rangle,
\end{aligned}
$$

where the common factor (log) that appears in Eqs. (4) and (38)-(40) is often taken to be

$$
(\log ) \equiv \ln \left(\frac{q^{2}}{a^{2}}\right) \approx \ln \left[\frac{\gamma^{2} \sigma_{v} \varepsilon_{h}}{r_{0} \beta_{h}}\right] .
$$

In the next section, we do numerical analyses using the ATF prototype damping ring to compare the Bane and CIMP high energy approximations to the more complete theory contained in Ref. [4]. Moreover, we compute the dependencies on bunch charge of the equilibrium transverse emittances, relative energy spread, and rms bunch length for the ATF, $\mathrm{ILC}_{\text {small }}$, and $\mathrm{ILC}_{\text {dogbone }}$ in order to check further the consistency of the Bane and CIMP high energy approximations.

\section{NUMERICAL COMPARISONS OF VARIOUS HIGH ENERGY APPROXIMATIONS}

To check the accuracy of the Bane and CIMP high energy approximations against the more complete theory contained in Ref. [4], we compute the growth times $T_{p}, T_{h}$, and $T_{v}$ for the ATF prototype damping ring described in Ref. [12]. For the nominal beam parameters contained in Table I, we average growth times around the damping ring lattice, yielding the numbers shown in Table II.

We see that the Bane and CIMP approximations are in excellent agreement with one another and in relatively good agreement with the more exact Ref. [4] results. Here, note again that Bane does not give a useful result for $1 / T_{v}$ for zero vertical dispersion, as is the case for this lattice.

Next, we compare the Bane and CIMP high energy approximations for the ATF [12], ILC $\operatorname{Ismall}_{\text {[13], and }}$ $\mathrm{ILC}_{\text {dogbone }}[14]$ damping rings by computing the detailed

TABLE I. Nominal beam parameters for the ATF prototype damping ring [12].

\begin{tabular}{lc}
\hline \hline Beam energy & $1.28 \mathrm{GeV}$ \\
Electrons per bunch & $10^{10}$ \\
Horizontal emittance & $1.15 \mathrm{~nm}$ \\
Vertical emittance & $4.03 \mathrm{pm}$ \\
Relative energy spread & $5.47 \times 10^{-4}$ \\
Bunch length (rms) & $5.59 \mathrm{~mm}$ \\
\hline \hline
\end{tabular}

TABLE II. Comparison of the Bane and CIMP high energy approximations to the more complete theory of Ref. [4] for the ATF prototype damping ring. Quantities are in $\sec ^{-1}$.

\begin{tabular}{lccc}
\hline \hline Approximation & $1 / T_{p}$ & $1 / T_{h}$ & $1 / T_{v}$ \\
\hline Ref. [4] & 390 & 267 & 9.10 \\
Bane & 435 & 291 & $\ldots$ \\
CIMP & 449 & 298 & 7.47 \\
\hline \hline
\end{tabular}

dependencies of equilibrium transverse emittances, relative energy spread, and rms bunch length on the number of electrons in each bunch. While IBS tends to increase these quantities, synchrotron radiation damping tends to decrease them, thus we wrote a computer code to find the various equilibrium values. At each bunch charge, one must average the growth times around the lattice, which takes the more complete theory in Ref. [4] many hours to complete using many popular software codes. Here we exploited the tremendous advantage of the high energy approximations, since the times for completing the computations were reduced from many hours to a few minutes. For each plot, we start with the nominal beam parameters shown in Table III.

Before presenting our results, a few words on betatron coupling are in order. In general, there are three principal contributions to the vertical emittance of an electron beam in a storage ring: a "direct" contribution, vertical dispersion, and betatron coupling. The direct contribution, which we will discuss later, is a small effect compared to the latter two and we ignore it for now. Also, there is some contribution from the vertical opening angle of the radiation, but for high energy beams, this is always small compared to the contributions from other sources. Collective effects other than IBS also may be important, but we do not consider those here. The effect of IBS on the vertical emittance depends upon whether the dominant contribution is from vertical dispersion or from betatron coupling.

If the vertical emittance is entirely dominated by vertical dispersion, the expressions given in Eqs. (11), (18), and (40) apply, and one can calculate the equilibrium vertical emittance $\varepsilon_{v}$ from

TABLE III. Nominal beam parameters for the ATF, ILC $\mathrm{small}_{\text {, }}$ and ILC $_{\text {dogbone }}$ damping rings.

\begin{tabular}{lccc}
\hline \hline \multicolumn{1}{c}{ Damping ring } & ATF & ILC $_{\text {small }}$ & ILC $_{\text {dogbone }}$ \\
\hline Beam energy $(\mathrm{GeV})$ & 1.28 & 5.066 & 5.00 \\
Relative energy spread $\left(\times 10^{-4}\right)$ & 5.47 & 15.1 & 12.9 \\
rms Bunch length $(\mathrm{mm})$ & 5.59 & 5.99 & 6.04 \\
Horizontal emittance $(\mathrm{nm})$ & 1.15 & 0.55 & 0.51 \\
Vertical emittance $(\mathrm{pm})$ & 4.03 & 1.41 & 1.29 \\
Horizontal damping time $(\mathrm{ms})$ & 17.86 & 26.75 & 27.86 \\
Vertical damping time $(\mathrm{ms})$ & 28.55 & 26.74 & 27.86 \\
Longitudinal damping time $(\mathrm{ms})$ & 20.38 & 13.37 & 13.93 \\
\hline \hline
\end{tabular}




$$
\varepsilon_{v}=\frac{T_{v}}{T_{v}-\tau_{v}} \varepsilon_{v 0}
$$

where $\tau_{v}$ is the vertical radiation damping time and $\varepsilon_{v 0}$ is the vertical emittance in the limit of zero bunch charge. However, if the vertical dispersion is zero (or negligibly small) throughout the ring, but there is some vertical emittance generated by betatron coupling, then we expect the horizontal IBS emittance growth to feed directly into the vertical plane. In the case that the vertical emittance is given entirely by betatron coupling, the relative growth from IBS in the vertical emittance will be the same as the relative growth in the horizontal emittance [15]

$$
\frac{\varepsilon_{v}}{\varepsilon_{v 0}}=\frac{\varepsilon_{h}}{\varepsilon_{h 0}} .
$$

If the vertical emittance is dominated by vertical dispersion and is much smaller than the horizontal emittance, the relative IBS emittance growth in the horizontal plane can be much larger than that in the vertical. Therefore, when calculating the IBS emittance growth, it is important to include some consideration of the relative contributions of dispersion and coupling to the vertical emittance. One approach is to proceed as follows. First, we write the vertical emittance in the limit of zero bunch charge as the sum of a contribution $\varepsilon_{v 0, \eta}$ from vertical dispersion and a contribution $\varepsilon_{v 0, \kappa}$ from betatron coupling

$$
\varepsilon_{v 0}=\varepsilon_{v 0, \eta}+\varepsilon_{v 0, \kappa} .
$$

For a given vertical dispersion, $\varepsilon_{v 0, \eta}$ may be estimated
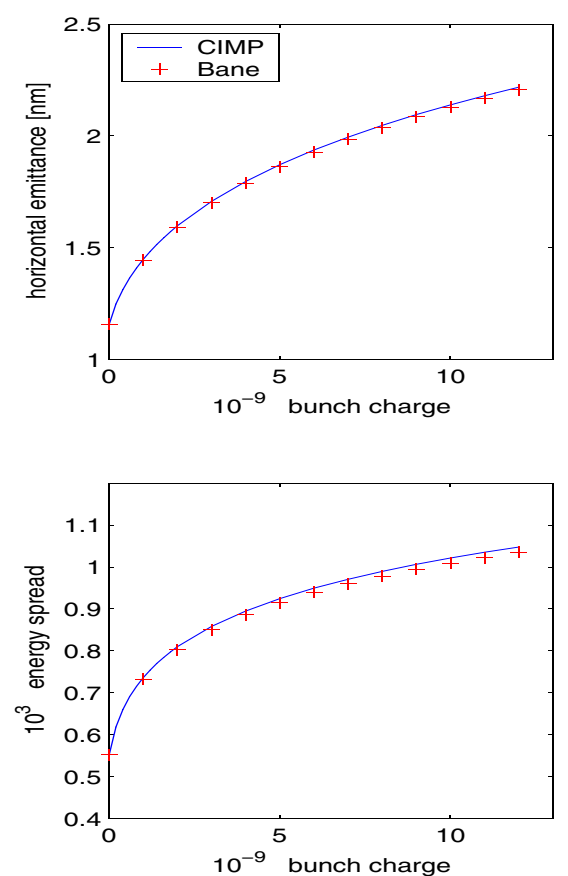

from [16]

$$
\varepsilon_{v 0, \eta} \approx J_{\epsilon}\left\langle\mathcal{H}_{v}\right\rangle \sigma_{p}^{2},
$$

where $J_{\epsilon}$ is the longitudinal damping partition, with a value typically between 1 and 2 . We then define the ratio $r_{\varepsilon}$

$$
r_{\varepsilon}=\frac{\varepsilon_{v 0, \kappa}}{\varepsilon_{v 0}}
$$

and finally express the vertical emittance in the presence of IBS as

$$
\varepsilon_{v}=\left[\left(1-r_{\varepsilon}\right) \frac{T_{v}}{T_{v}-\tau_{v}}+r_{\varepsilon} \frac{T_{h}}{T_{h}-\tau_{h}}\right] \varepsilon_{v 0} .
$$

Equation (47) is the equation for the vertical emittance growth used in our computer code. Of course, solution of the equilibrium distribution in the presence of IBS involves additional equations for the horizontal and longitudinal degrees of freedom.

For the ATF, the residual vertical dispersion at the lowest measured vertical emittance of $4 \mathrm{pm}$ has an rms value of about $1.5 \mathrm{~mm}$ [17], and we find that under these conditions, about $85 \%$ of the vertical emittance is generated by betatron coupling. We have assumed that the vertical emittances in the other lattices we consider will similarly be dominated by betatron coupling, and we expect to see the relative emittance growth from IBS in all three cases roughly equal in the horizontal and vertical planes.

Returning to our comparison of the Bane and CIMP high energy approximations, the results are shown in Figs. 1-3 for the $\mathrm{ATF}, \mathrm{ILC}_{\text {small }}$, and $\mathrm{ILC}_{\text {dogbone }}$ damping rings, re-
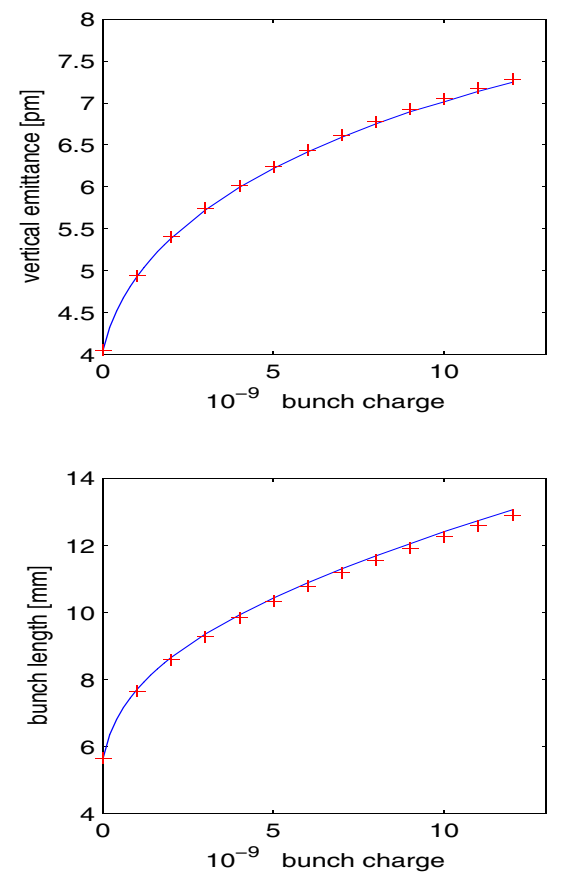

FIG. 1. (Color) Equilibrium transverse emittances, relative energy spread, and rms bunch length vs bunch charge for the ATF prototype damping ring. 

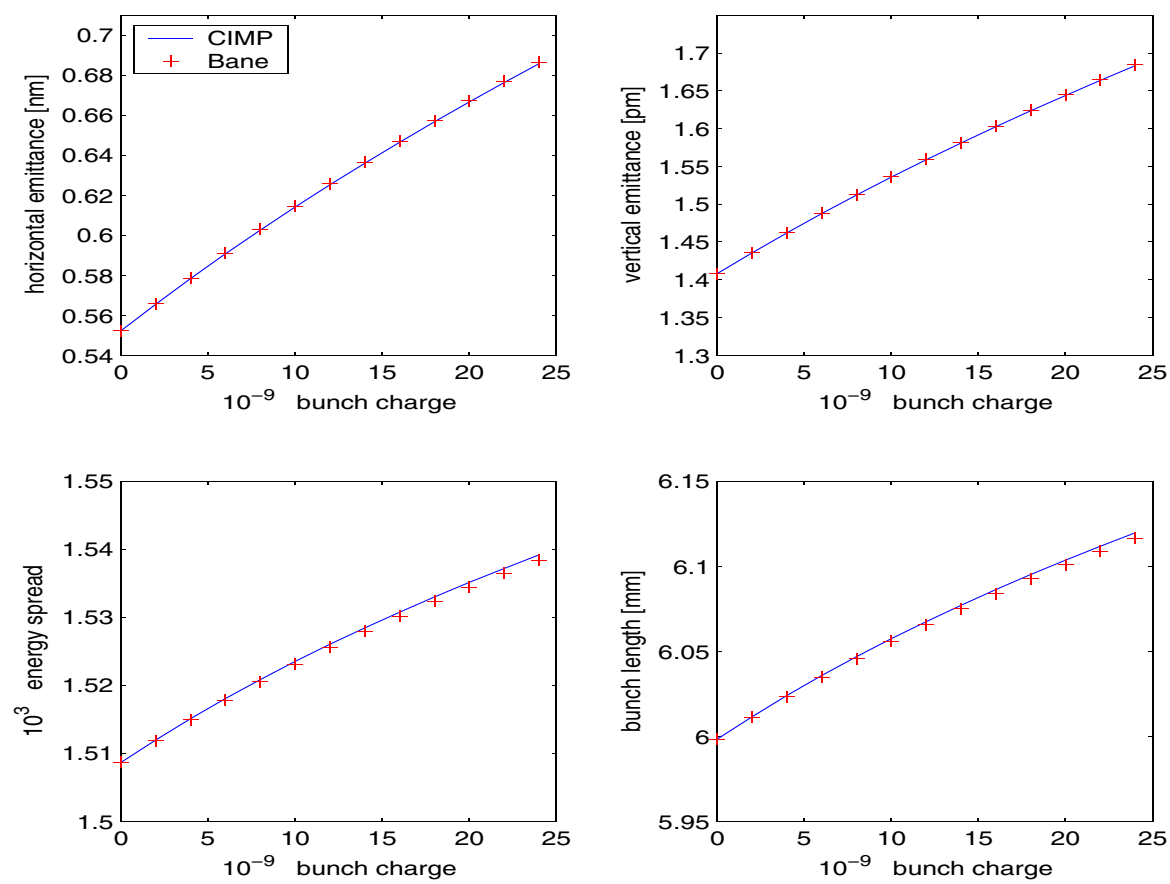

FIG. 2. (Color) Equilibrium transverse emittances, relative energy spread, and rms bunch length vs bunch charge for the $\mathrm{ILC}_{\text {small }}$ damping ring.

spectively. The Bane and CIMP approximations are in exceptional agreement with one another for all three damping rings. Moreover, the growths in the bunch dimensions calculated for the ATF are in good agreement with the experimental data reported by Honda et al. [17]. Note that the Coulomb log factor using Eq. (41) is $\sim 15$, which
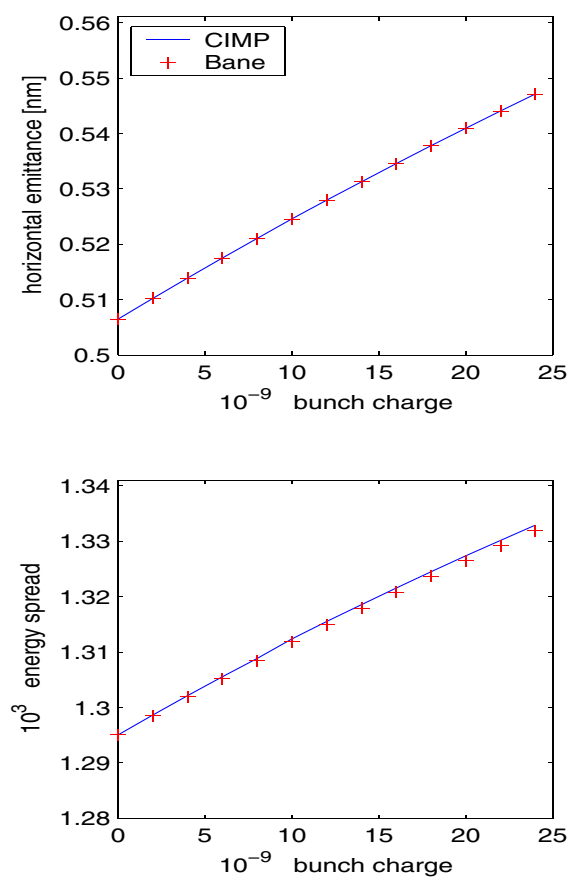

is somewhat larger than the value of $\sim 10$ as suggested in Ref. [18]. As discussed by Raubenheimer [9,19] and Kubo and Oide [18], this may reflect some non-Gaussian behavior of the beam.

Thus, in the presence of betatron coupling, we have shown explicitly that the Bane and CIMP high energy
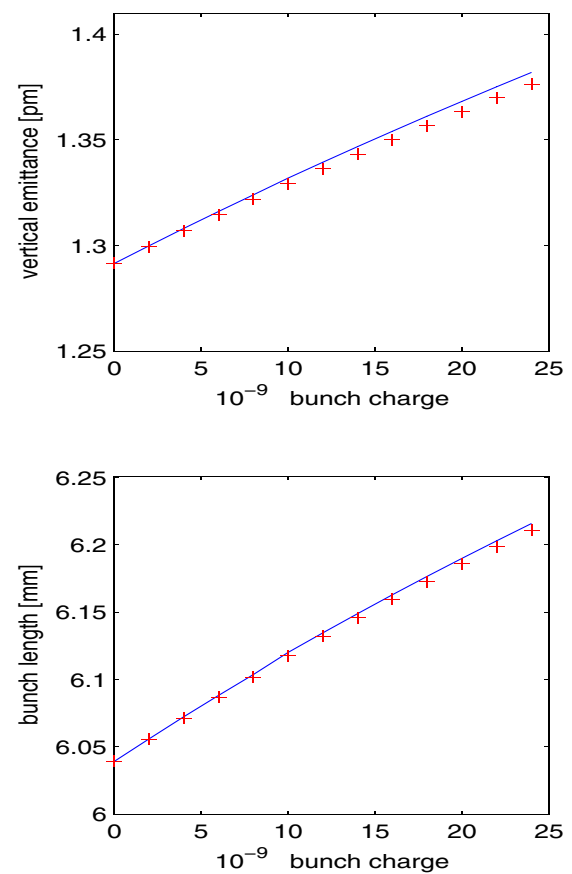

FIG. 3. (Color) Equilibrium transverse emittances, relative energy spread, and rms bunch length vs bunch charge for the ILC $_{\text {dogbone }}$ damping ring. 
TABLE IV. Comparison of the Bane and CIMP high energy approximations to the more complete theory of Ref. [4] for the ATF prototype damping ring without betatron coupling and with an rms vertical dispersion of $6.6 \mathrm{~mm}$. Quantities are in $\mathrm{sec}^{-1}$.

\begin{tabular}{lccc}
\hline \hline Approximation & $1 / T_{P}$ & $1 / T_{h}$ & $1 / T_{v}$ \\
\hline Ref. [4] & 325 & 238 & 239 \\
Bane & 369 & 257 & 263 \\
CIMP & 368 & 257 & 268 \\
\hline \hline
\end{tabular}

approximations are in excellent agreement with one another and give good agreement with the more complete theory of Ref. [4].

Finally, we use the ATF in order to examine the case of negligible betatron coupling and small, but nonzero, vertical dispersion so that Eq. (11) of the Bane approximation can be used. This should give us information as to the constraints of using Eq. (11). We maintain the nominal beam parameters of Table I; the necessary rms vertical dispersion is $6.6 \mathrm{~mm}$. Averaging around the ATF lattice gives the results contained in Table IV.

We see that the growth rates for the Bane and CIMP approximations are in excellent agreement with each other and both slightly overestimate the growth rates relative to the Ref. [4] results. After turning off the betatron coupling, the transverse growth rates have two contributions: a direct contribution, which was mentioned earlier, and a dispersion contribution. The direct contribution comes from the fact that two particles moving with zero transverse momentum, after collision, have some nonzero horizontal and vertical momentum. There is transverse emittance growth, even where the horizontal and vertical dispersion are both exactly zero. The transverse emittance growth from the direct contribution is rather slow, and it only dominates the vertical emittance growth for ultrasmall emittances, as we shall see.

The contribution to the emittance growth from the dispersion is generally larger than the direct contribution. The dispersion contribution arises from the change in longitudinal momentum in a collision, at a location where the dispersion is nonzero. This leads to an effective change in the transverse coordinates of the colliding particles, with respect to the off-energy orbits. The larger the dispersion, the faster the IBS emittance growth from this effect. Since the Bane approximation does not include the direct contribution to the IBS growth rates, Table IV shows that $6 \mathrm{~mm}$ is already a large enough vertical dispersion to completely dominate the direct contribution.

To see the differences between the Bane and CIMP approximations, we examine the ATF growth rates versus vertical emittance for ultrasmall vertical emittances. The results are shown in Fig. 4. Note that what is shown is not equilibrium solutions but just IBS growth rates versus vertical emittance, using the previous ATF parameters contained in Table I, but with different values of the vertical emittance.

There is good agreement between the Bane and CIMP approximations for the horizontal and longitudinal emittance growth rates over a wide range of vertical emittances; however, both approximations tend to overestimate the
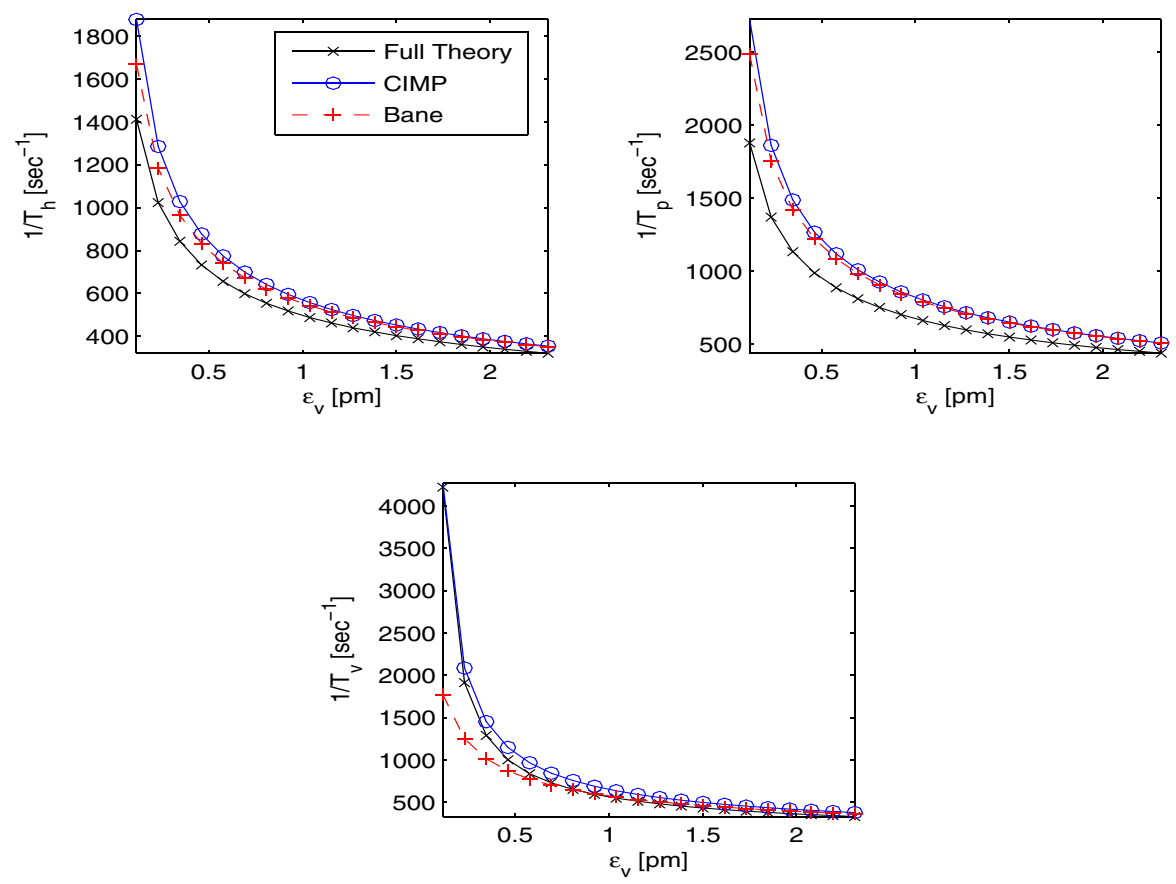

FIG. 4. (Color) IBS growth rates vs vertical emittance for the ATF prototype damping ring without betatron coupling. 
growth rates compared to the full theory. As the vertical emittance decreases, it is easy to see why the CIMP approximation tends to overestimate the horizontal and longitudinal growth rates. The approximation $\ln \left(q^{2} / a^{2}\right) \approx$ $\ln \left(q^{2} / b^{2}\right)$ becomes less good, and replacing $\ln \left(q^{2} / b^{2}\right)$ by $\ln \left(q^{2} / a^{2}\right)$ more and more contributes too much as the vertical emittance shrinks.

The growth rates for the vertical emittance are more interesting. Above about $0.5 \mathrm{pm}$, there is good agreement among all three methods. Below $0.5 \mathrm{pm}$, there is extremely good agreement between the full theory and the CIMP approximation; the Bane approximation significantly underestimates the vertical growth rate. This is the regime where the direct growth rate from IBS dominates, and the vertical dispersion is too small for the longitudinal growth rate to feed significantly into the vertical. Since the Bane approximation omits the direct contribution, it is not surprising that it underestimates the growth rate.

The best emittance achieved in the ATF is about $4 \mathrm{pm}$, so the regime where the Bane and CIMP approximations diverge is still about an order of magnitude below this. However, that vertical emittance regime is not completely unrealistic, since the theoretical minimum vertical emittance arising from the vertical opening angle of the radiation is about $0.08 \mathrm{pm}$.

It seems that the CIMP approximation appears to work well in all regimes: where the vertical emittance is dominated by the direct contribution, vertical dispersion, or betatron coupling.

\section{CONCLUSION}

We have derived completely integrated formulas for calculating the growth times of beam emittances due to intrabeam scattering at high beam energies. These formulas are based upon Bane's suggestion of how to modify the Piwinski theory to obtain one that includes varying accelerator lattice parameters. We have shown explicitly an equivalence between our modified Piwinski and Ref. [4] theories at high energies for accelerator lattices both with and without betatron coupling.

Also, we have demonstrated that a long-standing proportionality between transverse emittance and energy spread growth rates, which even predates the work of Bane, is not valid for calculating vertical IBS growth rates for ultrasmall vertical emittances in the absence of betatron coupling.

The use of high energy approximations greatly reduces the time for many calculations involving intrabeam scattering. Of course, before using any high energy approximation, it is always a good idea to check its validity against the more complete theory for a few representative cases to gain confidence in its accuracy.

\section{ACKNOWLEDGMENTS}

The authors appreciate helpful comments from Karl Bane. One of us (S. M.) appreciates the hospitality extended to him by William Barletta, Miguel Furman, and Andrzej Wolski during several visits to Lawrence Berkeley National Laboratory. This work was supported in part by the U.S. Department of Energy under Contract No. DEAC03-76SF00098. Further support was provided by two grants from the National Science Foundation, one subcontracted through Cornell University and the other subcontracted through Cooperative Agreement No. PHY0114343 with Hampton University's Center for the Study of the Origin and Structure of Matter.

[1] A. Piwinski, in Proceedings of the 9th International Conference on High Energy Accelerators, Stanford, CA, 1974 (SLAC, Stanford, 1974), p. 405.

[2] A. Piwinski, in Handbook of Accelerator Physics and Engineering, edited by A. Chao and M. Tigner (World Scientific, Singapore, 1999), p. 125.

[3] L. Evans and B. Zotter, CERN-SPS-80-15, 1980.

[4] J. Borken and S. Mtingwa, Part. Accel. 13, 115 (1983).

[5] M. Martini, CERN-PS-AA-84-9, 1984.

[6] G. Parzen, Nucl. Instrum. Methods Phys. Res., Sect. A 256, 231 (1987).

[7] S. Mtingwa and A. Tollestrup, Fermilab-Pub-89/224, 1987.

[8] J. Le Duff, in Proceedings of the CERN Accelerator School, Berlin, Germany, 1989, p. 114.

[9] T. Raubenheimer, Ph.D. thesis [SLAC-R-387, 1991], Sec. 2.3.1.

[10] J. Wei, in Proceedings of the Particle Accelerator Conference, Washington, DC, 1993 (IEEE, Piscataway, NJ, 1993), p. 3651.

[11] K. Bane, in Proceedings of the 8th European Particle Accelerator Conference, Paris, France, 2002 (EPS-IGA and CERN, Geneva, 2002), p. 1443.

[12] F. Hinode et al., KEK Internal Report No. 95-4, 1995.

[13] A. Xiao, "An Alternative TESLA Damping Ring Lattice Design at Fermilab, July 2004" (unpublished).

[14] "Technical Design Report for TESLA: The Superconducting Electron Positron Linear Collider with an Integrated X-Ray Laser Laboratory," edited by F. Richard, J.R. Schneider, D. Trines, and A. Wagner, DESY, 2001 (unpublished), Chap. 5.

[15] K. Bane et al., in Proceedings of the Particle Accelerator Conference, Chicago, IL, 2001 (IEEE, Piscataway, NJ, 2001), p. 2995.

[16] T. Raubenheimer, Ph.D. thesis [Report No. SLAC-R-387, 1991], Sec. 2.2.4.

[17] Y. Honda et al., Phys. Rev. Lett. 92, 054802 (2004).

[18] K. Kubo and K. Oide, Phys. Rev. ST Accel. Beams 4, 124401 (2001).

[19] T. Raubenheimer, Part. Accel. 45, 111 (1994). 